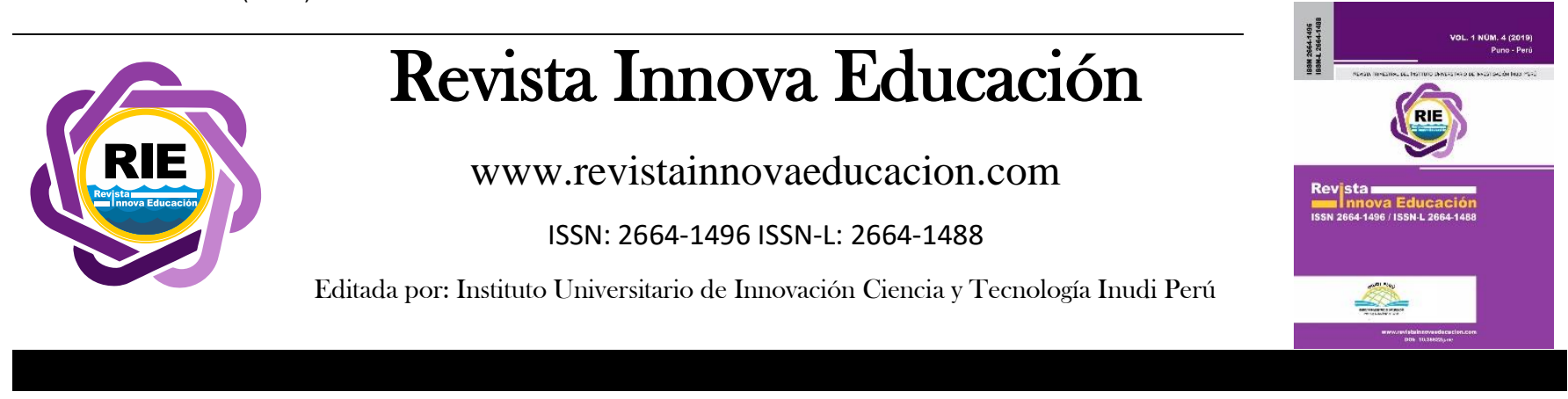

\title{
Procrastinación académica en el área de Física en estudiantes universitarios
}

\author{
Academic procrastination in the area of Physics in university students
}

\author{
Alí Morales ${ }^{1}$ \\ Universidad Nacional Experimental Politécnica de la Fuerza Armada, Caracas-Caracas, Venezuela \\ https://orcid.org/0000-0001-5419-6369
}

DOI: https://doi.org/10.35622/j.rie.2020.04.001

Recibido 30/08/2020/ Aceptado 21/09/2020

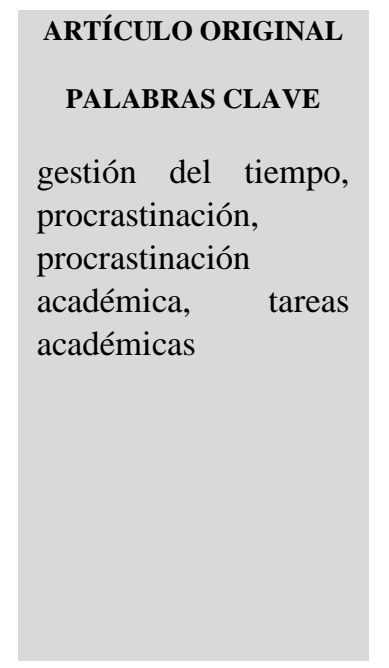

\section{KEYWORDS}

time management, procrastination, academic procrastination, academic tasks

\begin{abstract}
Con el objetivo de describir la procrastinación académica en el área de Física, de una población universitaria en Cojedes Venezuela, se realizó el presente estudio. Para su elaboración, se contó con alumnos correspondientes a la carrera Ingeniería de Telecomunicaciones de la Universidad Nacional Experimental Politécnica de la Fuerza Armada Núcleo Cojedes, cursantes de las asignaturas Física I y Física III. La metodología empleada fue un diseño no experimental, con investigación de campo y nivel descriptivo. La población fue de 43 estudiantes, con los que se usó en primera instancia como instrumento, una guía de observación. Para entrevistas y cuestionarios, se tomó una muestra de ocho estudiantes, correspondientes al 18,6\% de la población. Entre los resultados más relevantes, se encontró que más del $60 \%$ de los participantes presentaba conductas procrastinadoras. También se observó, que la mayoría se preparaban con poca antelación para las evaluaciones y manifestaban sentirse cómodos así, porque "trabajan mejor bajo presión". Como conclusión, se pudo verificar que existe presencia de procrastinación académica en la mayoría de la población, aunque se trata de una procrastinación activa.
\end{abstract}

In order to describe the academic procrastination in the Physics area of a university population in Cojedes Venezuela, the present study was carried out. For its preparation, there were students corresponding to the Telecommunications Engineering career of the National Polytechnic Experimental University of the Armed Forces Nucleus Cojedes, students of Physics I and Physics III subjects. The methodology used was a non-experimental design, with field research and descriptive level. The population was 43 students, with whom an observation guide was used in the first instance as an instrument. For interviews and questionnaires, a sample of eight students was taken, corresponding to $18.6 \%$ of the population. Among the most relevant results, it was found that more than $60 \%$ of the participants exhibited procrastinating behaviors. It was also observed that the majority prepared with little advance for the evaluations and stated that they felt comfortable this way, because "they work better under pressure". As a conclusion, it was possible to verify that there is a presence of academic procrastination in the majority of the population, although it is an active procrastination. 


\section{INTRODUCCIÓN}

El término procrastinación era bastante desconocido hasta hace poco; sin embargo, últimamente se ha ido dando a conocer a nivel mundial, sobre todo en la última década. Recientemente, se le ha ido prestando más atención desde el punto de vista científico, en dimensiones como la educativa, psicológica o clínica. Al realizar una revisión del término en la vigésimo tercera edición del Diccionario de la Lengua Española de la Real Academia (2014), se observa que procrastinar proviene del latín procrastināre: pro (a favor de) crastināre (relativo al día de mañana) y de su significado se menciona: "diferir, aplazar".

Sin embargo, el uso que se le da al término en la actualidad, tiene una significación un poco más compleja, por ejemplo, Díaz-Morales (2019) al referirse a la procrastinación, menciona que:

Consiste en la tendencia generalizada a aplazar el inicio y/o finalización de tareas planificadas para ser realizadas en un tiempo determinado. Tal tendencia a la postergación suele acompañarse de malestar subjetivo y no sólo es una cuestión de baja responsabilidad y gestión del tiempo, sino que supone un verdadero problema de auto-regulación a nivel cognitivo, afectivo y conductual. (p. 44)

Esta definición, es más ajustada a la procrastinación como problema y fenómeno de estudio, sobre todo desde el punto de vista educativo y psicológico.

Otro aspecto importante que se incluye en otras definiciones y que debe ser considerado, es el hecho de realizar una tarea generalmente más fácil y rápida, para sustituir la principal o la que debe realizarse. Al respecto, Rodríguez (2013) menciona que la procrastinación es "dejar de hacer lo que realmente tenemos que hacer y, en su lugar, hacer lo que no habría qué hacer, es decir, es dedicarse a lo secundario, a lo irrelevante o a pasar el rato" (p. 12), estas decisiones son irracionales y generalmente perjudiciales en distintos aspectos de la vida cotidiana.

Del mismo modo, existen otras definiciones que hacen énfasis en distintos aspectos que pueden ser objeto de estudio y permiten obtener nuevas dimensiones para investigaciones nacientes. Así, por ejemplo, Garzón y Gil (2017a) mencionan que "esta conducta implica posponer el inicio de una tarea, completarla en el último momento, sobrepasar los límites temporales fijados para la misma o incluso evitar indefinidamente su realización" (p. 2). Lo anterior, da entrada a un aspecto importante para delimitar la definición, como lo son los límites temporales o fechas límite, ya que una persona pudiera retrasar el hecho de comenzar una nueva carrera, aprender a tocar un instrumento musical, incluso leer un libro; sin embargo, son actividades que no tienen una fecha tope establecida y no quedan enmarcadas en la definición.

Con respecto a la prevalencia del fenómeno, existen numerosos datos; por ejemplo que del $20 \%$ al 25\% de la población general en América, son procrastinadores crónicos (Díaz-Morales, 2019) igualmente, hay estudios empíricos donde el fenómeno se asocia con variables académicas como en el caso de Tarazona et al. (2016), que determinaron que los estudiantes de lengua, 
literatura y comunicación estaban afectados en alto grado por procrastinación en un $71 \%$ o también que la misma correlaciona con la adicción al internet (Galarza et al., 2017)

A la presencia del fenómeno en el ámbito educativo, se le llama procrastinación académica, la cual consiste según Steel y Klingsieck (2016) en "el acto de posponer tareas o actividades académicas, de forma innecesaria, voluntaria y tras un intento decidido de finalizar las mismas, pese a esperar/predecir y experimentar un malestar subjetivo como causa del mismo" (p. 37). Se trata de un constructo inacabado, por lo que los estudios realizados sobre la misma van afinando esta definición, como muestran Barraza y Barraza (2018) al mencionar que se trata de "un dominio empírico que se encuentra en proceso de consolidación conceptual y, como tal, trae como consecuencia un componente metodológico, sobre todo en su aspecto instrumental, también en vías de consolidación” (p. 91).

La fecha límite mencionada previamente, tuvo un papel importante en el estudio de la procrastinación académica del presente trabajo, ya que, en función a esta, se describieron las conductas que provocaban los aplazamientos y ejecuciones en fechas cercanas a ese límite, los comportamientos posteriores a la entrega y también los efectos que tiene en las personas, como culpa, frustración, ansiedad o vergüenza (Neenan, 2008; García, 2009).

Existen cuatro modelos desde los cuales usualmente se ha enfocado el estudio de la procrastinación, estos son el psicodinámico, motivacional, conductual y cognitivo (Rothblum, 1990). En la presente investigación, en función de los objetivos planteados, se hizo énfasis en el conductual y motivacional. El primero, debido a que el estudio de los comportamientos que llevan a la entrega y realización de las actividades académicas en la fecha límite, es el punto de partida de la misma, de donde surgen los datos iniciales y el criterio establecido dentro de la definición. Respecto al segundo enfoque, se usó porque la persistencia en las conductas tiene un fuerte carácter motivacional, esto puede ser visto de dos formas; como problema, por constante procrastinación o como solución, porque según establece Cirino (2003) "la persona se mantiene realizando el esfuerzo por largos periodos de tiempo e insiste en ella hasta que logra el objetivo" (p. 81).

En Venezuela, la procrastinación académica ha sido poco investigada, existen algunos trabajos a nivel de pregrado en el área de psicología como por ejemplo el de González y Tovar (2015) y Coronado (2015) entre otros; a nivel de maestría destaca el de Bermúdez (2020) y a nivel doctoral la tesis de Morales (2019). Sin embargo, en las principales revistas científicas del área educativa y psicológica, así como a nivel de tesis doctorales en las principales universidades, buscadores académicos, bases de datos, repositorios y bibliotecas digitales, la revisión exhaustiva sobre el término arrojó escasos resultados.

Si lo anterior es comparado con otros países de Latinoamérica como Perú, Colombia o Ecuador, se observa una notable diferencia en cuanto a la cantidad de investigaciones y artículos científicos generados; pues en los países mencionados, se ha empezado a dar mayor importancia al tema en la última década. En este sentido, como problema que atañe a la parte educativa, hay una carencia en el estudio del fenómeno, donde trabajos como los previamente mencionados, reportan la presencia significativa de procrastinación académica en Venezuela a nivel universitario. 
En el contexto elegido para este estudio, se revisa el fenómeno de la procrastinación académica en un área específico (Física), lo que difiere con otras áreas por diferentes circunstancias, algunas de estas propias de una disciplina que requiere la aplicación de aproximaciones para explicar la realidad, conocimientos previos de matemática y cierto nivel de abstracción para la comprensión de los ejercicios. Otra circunstancia propia del contexto, es la importancia de las asignaturas correspondientes a esta área dentro del pensum de estudio, pues su reprobación implica no avanzar al siguiente semestre académico, lo que la diferencia en importancia con otras materias cursadas.

Para el presente estudio, se eligió como población a los estudiantes de Física I y Física III (no había matrícula en Física II) de la carrera Ingeniería de Telecomunicaciones del Núcleo Cojedes de la Universidad Nacional Experimental Politécnica de la Fuerza Armada (UNEFA), los cuales presentaban un alto número de reprobados cada semestre académico (por encima del 50\% en 2018 y 2019). Por supuesto, que los motivos de esto son multifactoriales, pero uno de estos posibles factores que se detectó y provocó una intención de investigación, fue el fenómeno de la procrastinación académica, pues un alto número de estudiantes solicitaba permanentemente prórroga para la entrega de las tareas y estos mismos en su mayoría, aparecían en el grupo de reprobados.

Inicialmente, mediante observación no sistemática, se fue revisando el fenómeno intentando ver el grado de presencia del mismo. Posteriormente, aún sin formalizar investigación alguna, se usó una guía de observación para revisar los tiempos de entrega de las distintas tareas asignadas, notando retrasos en la entrega de las mismas. Lo anterior, era solo un indicio de la presencia de procrastinación académica, pues el solo retraso en la entrega no bastaba para asegurarlo. Sin embargo, este primer contacto con el fenómeno, dio cabida al presente estudio, decidiendo realizar una investigación más formal, agregando que previamente no existían estudios en la universidad sobre el fenómeno en cuestión. Para plantear esta investigación, se tomaron en cuenta diversas dimensiones, como lo son la gestión del tiempo, inicio y culminación de tareas, funcionamiento bajo presión, preparación para los exámenes, actividades sustitutivas y malestar por procrastinar.

Como antecedentes, se tomaron como referencia dos investigaciones doctorales, que enfocan la procrastinación académica, dentro de distintas dimensiones que se asemejan al estudio realizado. En primera instancia, se usó la investigación de Natividad (2014) titulada "Análisis de la procrastinación en estudiantes universitarios" realizada en España. Respecto a ésta, el aporte estuvo en el estudio de la dimensión gestión del tiempo, que es una de las principales en ambos trabajos, así como el uso de técnicas como la entrevista.

En la misma línea de trabajo, se empleó la investigación de Barreto (2015) que lleva por nombre "Relajación en estados de ansiedad y procrastinación en ingresantes de la facultad de ciencias sociales" realizada en Perú, de la cual se tomó en cuenta el instrumento empleado para el registro de observaciones, similar al empleado en la presente, además de la coincidencia en las dimensiones psicológicas respecto al fenómeno, como el malestar por procrastinar. 
Se enmarcó teóricamente la investigación en dos teorías de entrada. La primera de ellas es la antigua teoría conductista de Thorndike (1911) de estímulo respuesta (Ley del efecto), asociándola con la conducta recompensada, esta ley menciona lo siguiente:

Entre las diversas respuestas emitidas ante una misma situación, las que están acompañadas o seguidas de cerca por satisfacción para el animal se conectarán más firmemente con la situación si las demás cosas son iguales, de modo que cuando la situación recurra tendrán más posibilidades de repetirse.

Las respuestas acompañadas o seguidas de cerca por malestar para el animal verán debilitadas sus conexiones con dicha situación si las demás cosas son iguales, de modo que cuando recurre la situación tendrán menos posibilidades de ocurrir. Mientras mayor sea la satisfacción o el malestar, mayor será el fortalecimiento o debilitamiento del vínculo. (p. 244)

Lo anterior, implica que cuando se recompensa una conexión entre estímulo y respuesta, la misma es reforzada conduciendo al aprendizaje y cuando es castigada, la conexión se debilita y termina por desaparecer. El uso de esta teoría, viene dado por utilizar el enfoque conductual de la procrastinación académica.

Del mismo modo, como segunda teoría de entrada se usó el modelo de procrastinación académica de Schraw et al. (2007), los cuales mencionan entre sus puntos que "la procrastinación puede llevar a consecuencias positivas o negativas en la calidad de vida; sin embargo, los alumnos no perciben el impacto en la calidad de su trabajo, entre otros aspectos" (p. 21). Todo esto se percibió en la forma como el fenómeno es percibido por los alumnos dentro de su entorno, además de que el modelo, toma en cuenta aspectos adaptativos asociados con el estudiar bajo presión.

El impacto de la investigación, se puede dilucidar en dos criterios específicos y plenamente intencionados, estos fueron el educativo y el psicológico. El primero de ellos, ya que las descripciones realizadas en el presente estudio, están basadas en la entrega de asignaciones o tareas y en los tiempos de preparación para evaluaciones académicas, por lo que los resultados obtenidos y las interpretaciones, se basan en el comportamiento de los estudiantes principalmente en el contexto educativo y la gestión que hacen del tiempo en ese ámbito.

Además, la presencia del fenómeno en situaciones de enseñanza aprendizaje, involucra a la didáctica, lo cual es asunto de importancia en cualquier nivel educativo, especialmente el universitario, donde el estudio podría servir de complemento, por ejemplo, en investigaciones sobre la deserción, que es uno de los problemas de carácter creciente en Venezuela en la educación superior, con incrementos significativos a partir del año 2015 (Albarrán, 2019).

En lo referente al ámbito psicológico, es de hacer notar que cuando se identifican ciertos comportamientos procrastinadores, algunos de estos están enmarcados en este aspecto, de allí la presencia de teorías de entrada como la de Thorndike. Del mismo modo, como se eligió el enfoque conductual como principal en el estudio, éste es materia de la psicología, recordando que se están estudiando comportamientos. Al final, la contribución en este sentido podría llegar de la mano de 
programas de intervención, que permitan tratar el fenómeno y conseguir resultados de interés educativo en dimensiones como el rendimiento académico, entre otras.

A partir de lo anterior, una vez puesta en contexto la situación observada de forma no sistemática inicialmente y luego sistematizada, además de presentar el posible impacto de la investigación, se plantean como interrogantes las siguientes ¿Cómo es la gestión el tiempo que realizan los estudiantes de Física de la UNEFA Cojedes, en la realización de tareas y preparación para los exámenes? ¿Qué aspectos permiten asegurar la presencia de procrastinación académica en estos estudiantes? ¿Cómo es la procrastinación académica presentada por los mismos? Para responder a esas interrogantes, se plantearon los siguientes objetivos de investigación: 1) Diagnosticar la gestión del tiempo en la entrega de tareas y preparación para los exámenes, en los estudiantes cursantes de Física, de la carrera ingeniería de telecomunicaciones de la UNEFA Cojedes. 2) Identificar los aspectos que permitan establecer la procrastinación académica en los estudiantes cursantes de Física, de esa población universitaria en Cojedes, Venezuela. 3) Describir la procrastinación académica en el área de Física, de esa población universitaria en Cojedes, Venezuela.

\section{MÉTODO Y MATERIALES}

En lo que se refiere a la metodología empleada en el presente estudio, se trató de un diseño no experimental (ex post facto). Inicialmente se diseñaron y aplicaron una serie de instrumentos, para observar el comportamiento de los estudiantes, pero éste no se manipuló ni se controló; sino que se recogió la información para luego ser analizada. Se observó sistemáticamente la situación presentada en cuanto a la entrega de las tareas y se preguntó mediante una entrevista, el tiempo de preparación para las evaluaciones.

A partir de lo anterior, la investigación se desarrolló hasta un nivel descriptivo, con lo cual se logra una clasificación de la información, en función de características comunes o incluso poniendo en relación los elementos observados, a fin de obtener la descripción (Hurtado, 2015). El trabajo presentado, puede llevarse a un nivel más avanzado, como el explicativo o correlacional, sin embargo, en este primer momento, se propone solo una descripción de los hechos, que ponga en evidencia situaciones procrastinadoras en los estudiantes, más allá de buscar explicaciones.

Como se mencionó anteriormente, la investigación se llevó a cabo en la Universidad Nacional Experimental Politécnica de la Fuerza Armada Nacional (UNEFA) con estudiantes de la carrera Ingeniería de Telecomunicaciones, cursantes de las asignaturas Física I y Física III (24 y 19 respectivamente, para un total de 43). Todos y cada uno de ellos, fueron sometidos a la técnica de observación sistemática; en ese sentido, la población estudiada fue la total, lo que significó que no hubo muestra para la aplicación del instrumento que fue una guía de observación.

Con respecto a las otras dos técnicas, fueron entrevista y encuesta. Antes de aplicar los instrumentos correspondientes, se seleccionó una muestra, para la cual se siguió el criterio señalado por Arias (2016) "En investigaciones descriptivas se recomienda seleccionar entre 10 y $20 \%$ de la población accesible" (p.87), bajo ese criterio, se seleccionaron cuatro estudiantes de cada grupo, para lo cual se utilizó un muestreo estratificado, dividiendo la población en subconjuntos cuyos elementos poseían características comunes (según el tiempo de entrega de la 
tarea), esto totalizó una muestra de ocho, lo cual representó el 18,6\% de la población total. Dado lo homogéneo del grupo en cuanto a la edad, la misma no se tomó en cuenta como factor para la selección de la muestra.

Sobre la aplicación del primer instrumento (guía de observación), se empleó para ver las principales características de los estudiantes, en cuanto al tiempo de entrega de las asignaciones, de allí se tomaron los datos a comparar con la fecha límite de entrega, que servirían en primera instancia como parte de la determinación de la procrastinación académica, complementando luego esta información con las entrevistas.

Además de esto, como segunda técnica se realizaron entrevistas semiestructuradas de respuestas abiertas, para lo cual se diseñaron guiones de entrevista que constaban de 14 ítems, aplicándolos tres veces durante el semestre académico a ocho alumnos por sesión, para totalizar 24. Finalmente, para la técnica de la encuesta, se diseñó un cuestionario de 19 ítems con respuestas policotómicas de escalamiento tipo Likert, con cinco categorías de respuestas asociadas a la frecuencia, las cuales iban desde "Siempre" hasta "Nunca".

La validez se realizó a través de tres expertos; un metodólogo por lo que podía aportar en la redacción de ítems, una psicóloga para revisión términos asociados al fenómeno y un docente del área de física, que conociera el grupo y su comportamiento. Se determinó la confiabilidad a través del coeficiente Alfa de Cronbach por tratarse de respuestas policotómicas, el cual arrojó un resultado de 0,87, que se traduce en una alta confiabilidad (Hernández et al., 2014). Este cuestionario se aplicó a ocho estudiantes al final de semestre, con la finalidad de contrastar los datos obtenidos de las entrevistas, que fueron la mayor fuente de información.

Con respecto a la temporalidad de los instrumentos aplicados, la misma se presenta en la siguiente figura:

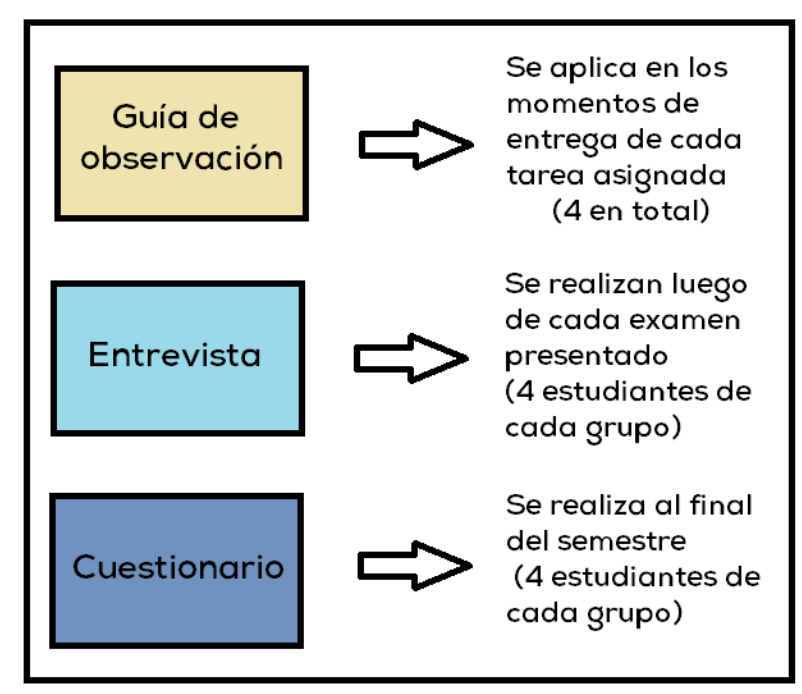

Figura 1. Momento de aplicación de los instrumentos usados en la investigación Fuente: El autor (2020) 
La estructura que se estableció para llevar a cabo la investigación, fue separar por fases el proceso de la misma. A continuación, se describe lo realizado en cada una de las fases:

Fase de preparación y planificación: En esta primera fase, se establecieron los parámetros que iban a utilizarse para poder hacer el diagnóstico de forma idónea. Para esto, se definió dentro del plan de evaluación de las asignaturas (Física I y Física III) las actividades que incluían exámenes, quices y lecturas a lo largo del período. Todo esto, incluía un cronograma de entrega de los informes de las lecturas, a lo largo de las 18 semanas que duraba el semestre académico, el cual se facilitó en la primera semana.

De la misma forma, se establecieron las fechas para las pruebas escritas (tres exámenes parciales y tres quices), planificando entrevistas estratégicas con estudiantes luego de los exámenes parciales, para determinar el tiempo en que se habían preparado para los mismos. Para todo esto, se diseñaron como instrumentos guiones de entrevista; en este sentido, las preguntas iban dirigidas a determinar si el alumno se preparaba con tiempo, además de los posibles factores que impedían tal preparación. Del mismo modo, se recogería en las entrevistas, información sobre el tiempo de realización de las tareas, para complementar lo obtenido del instrumento anterior.

Fase diagnóstica y de registro: En esta fase, se procedió a la administración de los instrumentos. Se comenzó con la guía de observación, donde se llevaba lo referente a los tiempos de entrega de las actividades asignadas (lecturas), contrastando contra la fecha límite establecida en el cronograma del plan de evaluación, se utilizó un instrumento por cada entrega de informes sobre las lecturas asignadas.

Para la verificación y contrastación de los tiempos de anticipación con que se realizaban las tareas, así como también para el registro de los tiempos de preparación para los exámenes y los factores que influían en ellos, se realizaron entrevistas a cuatro estudiantes por grupo tras cada examen presentado, variándolos para cada medición; fueron ocho entrevistas por cada examen parcial, para totalizar 24 a lo largo del semestre. Finalmente, se aplicó un cuestionario con el objetivo de verificar y contrastar los datos de las entrevistas, este se administró al final del semestre académico a una muestra de la población, que igualmente fue de ocho.

Fase de revisión: Una vez finalizado el semestre académico, se procedió a la recopilación y tabulación de toda la información, comparando las fechas de entrega con los resultados obtenidos, distinguiendo la cantidad de estudiantes que entregaban con retardo y los que hacían con alguna antelación las asignaciones, luego se procedió con la totalización de datos. Para el análisis estadístico de los cuestionarios, se utilizó estadística descriptiva. Finalmente, se revisó profundamente lo obtenido de las entrevistas, buscando elementos que pudieran servir para complementar la descripción final de la procrastinación académica.

Fase de descripción: En esta etapa, hay que destacar que se procedió a describir con detalle, cada una de las situaciones reflejadas en los distintos instrumentos utilizados en la segunda fase y revisados en la tercera, detallando de la forma más pormenorizada posible y teniendo en cuenta las dimensiones previamente establecidas. Lo mismo se hizo con las teorías de entrada para cada uno de los comportamientos y situaciones relacionadas con la procrastinación de los estudiantes, 
tanto en la entrega de los informes de las lecturas, así como también en la gestión que hicieron del tiempo, a la hora de presentar las evaluaciones.

\section{RESULTADOS}

Los resultados de la guía de observación usada para registrar la entrega de las tareas (informes de las lecturas), se clasificaron en: Entregados con anticipación, Entregados en la fecha límite, Solicitud de prórroga (manifestaron alguna razón para no entregar en la fecha establecida) y No entregados. Los porcentajes de informes entregados por lectura, de los estudiantes de Física I y Física III se reflejan en las tablas 1 y 2 respectivamente (la asignatura Física II no tuvo matrícula):

\section{Tabla 1.}

Porcentaje de informes entregados por lecturas realizadas, respecto a la fecha límite. Física I.

\begin{tabular}{lcccc} 
INFORMES ENTREGADOS & L1 & L2 & L3 & L4 \\
\hline Entregados con anticipación. & $12.5 \%$ & $8.3 \%$ & $12.5 \%$ & $0 \%$ \\
Entregados en la fecha límite. & $75 \%$ & $75 \%$ & $70.8 \%$ & $66.6 \%$ \\
Solicitud de prórroga & $4.2 \%$ & $8.3 \%$ & $0 \%$ & $16.7 \%$ \\
No entregados. & $8.3 \%$ & $8.3 \%$ & $16.7 \%$ & $16.7 \%$ \\
\hline
\end{tabular}

Nota. L1, L2, L3y L4 corresponden a cada lectura realizada durante el semestre.

Se consideró como entregado con anticipación, incluso un día antes de la fecha límite.

Fuente: El autor (2020)

Tabla 2.

Porcentaje de informes entregados por lecturas realizadas, respecto a la fecha límite. Física III.

\begin{tabular}{lrrrc} 
INFORMES ENTREGADOS & L1 & L2 & \multicolumn{1}{c}{ L3 } & L4 \\
\hline Entregados con anticipación. & $10.5 \%$ & $5.3 \%$ & $5.3 \%$ & $0 \%$ \\
Entregados en la fecha límite. & $68.4 \%$ & $73.7 \%$ & $78.9 \%$ & $57.9 \%$ \\
Solicitud de prórroga & $15.8 \%$ & $10.5 \%$ & $0 \%$ & $10.5 \%$ \\
No entregados. & $5.3 \%$ & $10.5 \%$ & $15.8 \%$ & $31.6 \%$ \\
\hline
\end{tabular}

Nota. L1, L2, L3y L4 corresponden a cada lectura realizada durante el semestre.

Se consideró como entregado con anticipación, incluso un día antes de la fecha límite.

Fuente: El autor (2020)

Sobre los hallazgos obtenidos para estas dos primeras tablas, se observa en primera instancia, que los estudiantes se manejan en función a la fecha límite, más allá de los posibles adelantos que puedan realizar en las tareas. Estos resultados por sí solos, no arrojaron valores elevados de procrastinación académica, salvo por el 15.8\% que solicitó prórroga.

Los comportamientos mostrados, estuvieron en concordancia con el modelo de Schraw et al. (2007) en uno de sus cinco aspectos, el cual menciona que dejan para última hora las actividades académicas, debido a que suponen que es adaptativo y eficiente, además de considerarlo parte de un repertorio flexible de estrategias de afrontamiento cognitivas. Con respecto al aumento de porcentaje en el tiempo de los informes no entregados, posteriormente en las entrevistas se obtuvo 
que se debía a que algunos estudiantes hacia el final del período, ya sentían que iban a reprobar la asignatura y dejaban de entregar las tareas.

En el mismo orden de ideas, durante las entrevistas semiestructuradas realizadas a los estudiantes, se recogieron datos interesantes en cuanto a los días de anticipación para realizar las tareas y los tiempos de preparación para los exámenes, además de los motivos de ese tiempo, entre otros. Algunos resultados (los más significativos) de las respuestas de las 24 entrevistas realizadas a lo largo del semestre académico, se recogen en las tablas 3,4 y 5, aclarando que las preguntas eran abiertas y las respuestas fueron tabuladas.

Tabla 3.

¿Con cuántos días de anticipación realizaste la tarea?

\begin{tabular}{|c|c|c|c|c|c|c|}
\hline Respuestas & $\begin{array}{l}\text { Cinco o } \\
\text { más días }\end{array}$ & Tres días & Dos días & Un día & Mismo día & $\begin{array}{l}\text { No la } \\
\text { realicé }\end{array}$ \\
\hline Frecuencia & 3 & 3 & 7 & 6 & 2 & 3 \\
\hline Porcentaje (\%) & 12.5 & 12.5 & 29.1 & 25.0 & 8.3 & 12.5 \\
\hline
\end{tabular}

Nota. La información fue tabulada a partir de las entrevistas.

Fuente: El autor (2020)

La inclusión de esta pregunta dentro de la investigación, fue clave para poder contrastar contra lo obtenido a través de la guía de observación. Se observó, que un 62.4\% realizaba la tarea con dos o menos días de anticipación respecto de la fecha límite (número razonablemente cerca de esta fecha), lo que podría aumentar si se considera que los que no la realizaron, manifestaron la intención de hacerla, pero presentaron excusas; incluso, el grupo de tres días antes que no se incluyó en la cuenta, pudiera ser parte también de las cifras, llevando los porcentajes por encima del $80 \%$.

Por sí solos, estos resultados ahora si dan muestras de procrastinación académica, pero lo interesante es que, al ser comparados con lo obtenido previamente con la guía de observación, se nota que un número importante de procrastinadores, sí entregan la tarea a tiempo, aunque la hagan al borde de la fecha límite. Todo esto, pudiera incluir a este último grupo dentro de lo que Chu y Choi (2005) denominan procrastinadores activos, que son aquellos que prefieren trabajar bajo presión y tomar decisiones deliberadas para posponer las cosas. Los autores, refieren además que estos últimos, comparten características con los no procrastinadores, en cuanto a percepción de autoeficacia y los estilos de afrontamiento.

Tabla 4.

¿Con cuántos días de anticipación estudiaste para la evaluación presentada (examen)?

\begin{tabular}{lcccccc}
\hline \multicolumn{1}{c}{ Respuestas } & Siete días & Cinco días & Tres días & Dos días & Un día & Mismo día \\
\hline Frecuencia & 2 & 1 & 6 & 8 & 6 & 1 \\
Porcentaje $(\%)$ & 8.3 & 4.2 & 25.0 & 33.3 & 25.0 & 4.2 \\
\hline
\end{tabular}

Nota. La información fue tabulada a partir de las entrevistas.

Fuente: El autor (2020) 
Con respecto a las evaluaciones presentadas, si se toma el mismo parámetro de dos días de anticipación, para examinar la preparación para las evaluaciones con retardo (que incluso pudiera aumentarse), curiosamente aparece el mismo porcentaje de $62.4 \%$ de estudiantes dentro de ese rango (la suma de cada estrato en los dos casos, da 15), lo cual viene a decir que bajo ese criterio, los estudiantes de esta población, presentan similar procrastinación en exámenes que en tareas.

Estos números, parecieran contradecir algunas respuestas de los estudiantes en las entrevistas, donde mencionan que las tareas les quitan tiempo de estudio para los exámenes, lo que haría que el número de este último aspecto fuese menor, pero no ocurre tal cosa. Del mismo modo, en lo obtenido en la contrastación con la pregunta anterior, se nota que la similitud de los porcentajes entre actividades ya fue reportada por Onwuegbuzie (2004) aunque con valores menores, ya que obtuvo $41.7 \%$ de procrastinación para las tareas escritas y $39.3 \%$ para los exámenes.

Tabla 5.

¿Qué actividades haces en el tiempo en que deberías prepararte para los exámenes?

\begin{tabular}{lcccccc} 
Respuestas & $\begin{array}{c}\text { Navegar } \\
\text { en internet }\end{array}$ & $\begin{array}{c}\text { Descansar } \\
\text { o dormir }\end{array}$ & $\begin{array}{c}\text { Chatear } \\
\text { por el } \\
\text { teléfono }\end{array}$ & $\begin{array}{c}\text { Ver TV- } \\
\text { Películas en } \\
\text { PC }\end{array}$ & Salir & Otra \\
\hline Frecuencia & 4 & 5 & 6 & 3 & 4 & 2 \\
\hline Porcentaje (\%) & 16.7 & 20.8 & 25.0 & 12.5 & 16.7 & 8.3 \\
\hline
\end{tabular}

Nota. La información fue tabulada a partir de las entrevistas.

Fuente: El autor (2020)

Autores como Steel (2011) consideran que "la procrastinación en si misma se encuentra en la decisión de lo que se hará y no en la postergación en sí” (p. 21); en este sentido, la toma de esa decisión se hace entre lo que debe realizarse y alguna otra actividad. Para la correcta descripción del fenómeno, este tipo de actividad alterna debe ser considerada. En ese sentido, en la tabla anterior, se observa que la tecnología, es factor preponderante elegido como tarea sustitutiva de la principal o como distracción, con más de un 54\%. Algunos estudios, confirman la relación de la tecnología con la procrastinación, como Castro y Mahamud (2017) que reportaron "que existe una relación positiva y significativa entre procrastinación académica y adicción a internet $(\mathrm{r}=0.322$; p < 0.05)" (p. 194) aunque en el caso estudiado, no se manejen datos de adicción.

Tabla 6.

Si te preparas con tres días o menos de anticipación ¿Por qué motivo lo haces de esa manera?

\begin{tabular}{lccccc}
\hline Respuestas & $\begin{array}{c}\text { No me da } \\
\text { tiempo antes }\end{array}$ & $\begin{array}{c}\text { Desgaste por } \\
\text { traslado } ~ \\
\text { Cansancio }\end{array}$ & $\begin{array}{c}\text { Funciono } \\
\text { mejor bajo } \\
\text { presión }\end{array}$ & $\begin{array}{c}\text { No entiendo } \\
\text { la asignatura }\end{array}$ & Otra \\
\hline Frecuencia & 3 & 4 & 12 & 3 & 2 \\
Porcentaje $(\%)$ & 14.29 & 19.05 & 50 & 14.29 & 9.52 \\
\hline
\end{tabular}

Nota. La información fue tabulada a partir de las entrevistas.

Fuente: El autor (2020)

Esta pregunta también fue una contrastación con lo obtenido en el primer instrumento. En el análisis de las dos primeras tablas, se mencionó como posible explicación de lo obtenido, que uno de los cinco aspectos del modelo de Schraw et al. (2007) mencionaba que los estudiantes dejan para última hora las actividades académicas, debido a que suponen que es adaptativo y eficiente, 
en esta tabla se ratifica lo mencionado, observando que la mitad de ellos percibe que funciona mejor bajo presión.

Para tener la percepción anterior, hay que decir que los alumnos, deben haber obtenido resultados satisfactorios con estos comportamientos, puesto que sienten que su funcionamiento es mejor. Esto, pudiera deberse a conductas reforzadas positivamente, según lo expuesto previamente de la ley de efecto de Thorndike, ya que, si el estudiante procrastinó, pero de igual forma sintió que obtuvo un resultado acorde a sus expectativas, dicha satisfacción le refuerza ese comportamiento.

Finalmente, se muestran resultados relevantes del último instrumento (cuestionario), que contaba con 19 ítems politómicos con escalamiento tipo Likert, el cual se aplicó a final de semestre académico, también a ocho estudiantes. Hay que mencionar, que este instrumento es secundario dentro del presente estudio, pues busca contrastar y complementar lo obtenido a partir de las entrevistas realizadas, que fueron las que brindaron la mayor parte de la información para la descripción del fenómeno. Los resultados del instrumento en cuestión, se presentan en las siguientes tablas:

\section{Tabla 7.}

Luego de finalizada una evaluación de la asignatura ¿sientes que pudiste o debiste prepararte mejor?

\begin{tabular}{lccccc}
\hline Alternativas & Siempre & $\begin{array}{c}\text { Casi } \\
\text { siempre }\end{array}$ & $\begin{array}{c}\text { Algunas } \\
\text { veces }\end{array}$ & Casi nunca & Nunca \\
\hline Frecuencia & 6 & 1 & 1 & 0 & 0 \\
Porcentaje $(\%)$ & 75.0 & 12.5 & 12.5 & 0.0 & 0.0 \\
\hline Fuente
\end{tabular}

Fuente: El autor (2020)

En distintas definiciones de la procrastinación académica, se habla sobre el aplazamiento de las tareas; sin embargo, Gil et al. (2020) mencionan que este "es un componente necesario, pero no suficiente para definir la procrastinación. Este retraso se considera procrastinación cuando va unido a malestar o consecuencias negativas para los individuos que lo practican" (p. 184). Los resultados de la tabla, muestran que el $75 \%$ estudiantes, sienten que pudieron alistarse mejor para los exámenes; es decir, que algo no estuvo bien al momento de prepararse, lo que permite mencionar que el retraso para este tipo de evaluación, generó cierta conducta de malestar que se asocia con el fenómeno estudiado.

Tabla 8.

¿El tiempo libre que tienes en tu estancia en la universidad lo inviertes actividades distintas a estudiar?

\begin{tabular}{lccccc}
\multicolumn{1}{c}{ Alternativas } & Siempre & $\begin{array}{c}\text { Casi } \\
\text { siempre }\end{array}$ & $\begin{array}{c}\text { Algunas } \\
\text { veces }\end{array}$ & Casi nunca & Nunca \\
\hline Frecuencia & 1 & 3 & 4 & 0 & 0 \\
Porcentaje $(\%)$ & 12.5 & 37.5 & 50.0 & 0.0 & 0.0 \\
\hline
\end{tabular}

Fuente: El autor (2020)

Respecto a esta última tabla, es conveniente aclarar que esta variable es muy particular de esta población, porque los horarios de estudios de esta carrera, no se presentan en bloques de un 
solo turno, sino que la mayor parte del día, los alumnos lo pasan en la universidad, teniendo espacios libres de seis horas y hasta más. Esto, disminuye su permanencia en el hogar para estudiar con normalidad, situación que se presenta incluso los días sábados.

En este sentido, el aprovechamiento del tiempo en las instalaciones de la universidad, podría ser positivo para un mejor desempeño académico, pues pudieran gestionar su tiempo allí entre espacios de ocio, socialización o deportes y aun así, poder dedicar tiempo al estudio. Sin embargo, los resultados muestran que el 50\% tiende a realizar actividades distintas de estudiar en este tiempo y el restante $50 \%$, sólo algunas veces se prepara o realiza tareas en esos espacios, dentro del recinto universitario.

La importancia de este hallazgo, más allá de las particularidades de la población de estudio, es que no se consiguieron antecedentes en el tratamiento de esta variable en otras investigaciones, lo cual podría crear un precedente que sirva de comparación, con otra población con características similares y cuyo comportamiento, haya pasado desapercibido por otros investigadores.

\section{DISCUSIÓN DE RESULTADOS}

En principio, se pudo observar que a pesar de que los alumnos contaban desde la primera semana, con un cronograma de entrega de tareas, en promedio menos del 10\% las entregó con anticipación y aunque un 70\% aproximadamente en promedio, siempre la entregó a tiempo, lo hicieron en la fecha límite. Las tareas se asignaron la primera semana de clase y la primera entrega correspondía a la cuarta, por lo que el tiempo para la realización de las mismas fue bastante amplio y no se requería conocimiento previo ni del cursado en el período. Incluso, se presentó el caso de un estudiante de Física I, que en la segunda semana entregó las primeras tres tareas.

Autores como Tuckman (2002) sugieren como medida para combatir la procrastinación, un mayor tiempo para la realización de las tareas; sin embargo, los resultados del presente estudio insinúan que no es un factor relevante, pues como también reportaron Naturil et al. (2018) en España, en su estudio obtuvieron porcentajes de procrastinación superiores al 80\%, a pesar de tener un tiempo amplio para elaborar las tareas.

De los estudiantes que no entregaron los informes; se puede alegar dos cosas, que no tuvieron intención de hacerla o que demoraron tanto, que al final no la entregaron. Si se trata de lo primero, no puede ser calificada como procrastinación académica, pues al no mostrar intenciones de realizarla, no entran dentro de la definición del fenómeno. En caso de ser lo segundo, si formarían parte de la estadística de los que presentan el comportamiento, pues retrasaron tanto la entrega, que al final no la entregaron; este último caso formaría parte de los llamados procrastinadores pasivos (Chu y Choi, 2005).

Un aspecto a tener en cuenta, es que los estudiantes de Física III (curso más avanzado) presentaron mayores conductas procrastinadoras, ya que en porcentaje entregaron menos veces anticipadamente, dejaron de entregar más veces y solicitaron más prórrogas que los de Física I. Esto coincide con lo encontrado por Somers (2008) que mencionó que el fenómeno, se acentúa en los últimos años de carrera, antes de obtener el grado universitario. Por contraste, un estudio 
empírico realizado por Rodríguez y Clariana (2017) mostró que la procrastinación no depende del curso académico en el que el estudiante esté matriculado.

Con respecto a las pruebas escritas presentadas; en las entrevistas realizadas, se obtuvo que un porcentaje mayor al 60\%, se preparan para estas como máximo dos días antes y muchas veces el día anterior, con lo que los tiempos de preparación no son los adecuados para lo largo y complejo de los contenidos; así que se acumulan los temas y se hace complicado estudiarlos. Sin embargo, luego de presentadas las evaluaciones, los estudiantes sienten que pudieron obtener mejores resultados, situación de inconformidad mencionada previamente.

A pesar de lo anterior, estudios empíricos como el de Domínguez y Campos (2017) reportaron que, el hecho de que el estudiante cumpla a tiempo (o no), con las labores encomendadas o se organice para hacer frente a las exigencias académicas de las asignaturas, no depende de la satisfacción experimentada con su forma de estudiar. Sin embargo, en el proceso de las entrevistas, varios de ellos manifestaron que cuando están ante la misma situación, vuelven a repetir los comportamientos, conduciendo esto, a una especie de "circulo vicioso" que puede llevar a una mayor procrastinación (Garzón y Gil, 2017b).

También se notó en los resultados, que el uso de tecnología para actividades distintas a la académica, resta tiempo al alumno que antepone muchas veces chatear, navegar, revisar redes sociales, ver televisión o películas al acto de estudiar; esto, coincide con lo reportado por Condori y Mamani (2016), que sugieren que "los estudiantes con niveles altos de procrastinación, utilizan la mayoría de su tiempo libre en la navegación de redes sociales, chats, y otros lugares del ciberespacio que ofrecen actividades placenteras" (p. 277). Estudios empíricos previos, como el de Matalinares et al. (2017), obtuvieron por ejemplo a través de un análisis correlacional, un resultado significativo y positivo en la relación de las variables de procrastinación y adicción a redes sociales, situación esta última asociada a la tecnología, aunque no se pueda hablar de adicción para la población de estudio, pues no existen investigaciones al respecto en ella.

Otro aspecto destacado, aunque no nuevo dadas las revisiones de los antecedentes previos, es la respuesta de los alumnos que mencionan que les va mejor o se sienten mejor estudiando bajo presión, que cuando el examen no está cerca; pues en este último caso, no se concentran o no sienten ánimo para prepararse. Este comportamiento procrastinador, lo presentan más de la mitad de los consultados, lo cual se encuentra acorde con el modelo de Schraw et al. (2007) como se mencionó en el apartado de resultados.

Respecto al modelo mencionado previamente, existe otro de sus aspectos que también se relaciona con el comportamiento de los estudiantes referido a estudiar bajo presión y es el que menciona que, aunque el mismo puede llevar a consecuencias positivas o negativas en la calidad de vida; los alumnos reportan que no tiene casi impacto en la calidad de su trabajo. Esto, explicaría porque adoptan esta conducta, pues optimizarían los tiempos sin afectar significativamente sus calificaciones. Sin embargo, estudios empíricos como el de Gil et al. (2020) mencionan en sus resultados que, de acuerdo con los estadísticos descriptivos obtenidos por ellos respecto a los motivos del alumnado para procrastinar, que "el deseo de sentir la presión de que el tiempo se acaba y asumir el reto de realizar la tarea en el último momento, es algo que no mueve al alumnado a procrastinar" (p. 192). 
En lo que respecta a la gestión del tiempo, también se observa que a pesar de que el alumno pasa aproximadamente entre seis y nueve horas diarias en las instalaciones de la universidad, el tiempo en el que no tiene clases (que muchas veces es más de la mitad), lo invierte poco en estudio, como lo muestra la tendencia positiva obtenida, hacia actividades distintas a la de estudiar. En las entrevistas, los alumnos mencionan que dejan para estudiar al llegar a casa, pero entonces son propensos a factores como la fatiga, la tecnología como factor extracadémico o salir a divertirse.

Lo anteriormente mencionado, fue un hallazgo muy particular de la presente investigación, como elemento de la gestión del tiempo (dentro de la universidad) del que no se consiguió presencia en otros trabajos y se hace presente por los horarios complicados que se manejan en la carrera específica del estudio (Ingeniería de Telecomunicaciones) para la población de trabajo, que obligan a permanecer al alumno muchas horas en las instalaciones de la universidad, desaprovechando los espacios libres que pudieran ser productivos para la preparación académica y realización de tareas. Por otra parte, no hay que perder de vista que los resultados obtenidos están centrados en un área específica (Física), una variable moderadora que hace particular al estudio y cuyo tipo, es influyente en los efectos medidos, entre otras causas porque esa área es percibida como difícil por los estudiantes, según lo mencionado en las entrevistas.

Con lo obtenido en este opúsculo, se pretende abrir la puerta a una serie de investigaciones en esta misma vía, hasta conseguir explicaciones del porqué ocurre el mismo, conseguir su correlación por ejemplo con el rendimiento académico o con la deserción escolar, donde existen claros indicios que es un factor importante (Garzón y Gil 2017b). También, se pretende profundizar en próximos estudios en variables como el tiempo semanal invertido en la asignatura y el tiempo de estudio dentro de las instalaciones de la universidad, lo cual también puede contribuir significativamente a nuevos hallazgos, en el campo de la procrastinación académica.

Con respecto al alcance del estudio, hay que decir que la procrastinación académica es multidimensional y se quedaron fuera de la investigación, variables importantes como el sexo, el curso académico y la influencia del docente, entre otras significativas que limitan los resultados obtenidos. Este trabajo, es un inicio en el estudio de la procrastinación en la institución y como ya se vio, existe muy poco al respecto incluso en el país. Igualmente, en cuanto al cuestionario, su uso fue de complemento de la información, pues la mayor parte de lo obtenido vino de las entrevistas realizadas, por lo que no se aplicó a toda la población si no a la muestra seleccionada.

Referido a la utilidad de los resultados, hay que recordar que la procrastinación académica es un constructo inacabado y existen aún muchas discrepancias en su definición, por lo que distintos aportes ayudan a fortalecer este panorama. Por ejemplo, el hallazgo de que los largos tiempos para la realización de las tareas, no disminuyen la procrastinación significativamente, da cabida a estudios sobre el tiempo idóneo, en el cual el alumno se sienta menos afectado por el fenómeno. Del mismo modo, sobre la gestión del tiempo dentro de las instalaciones de la universidad cuando es largo, que es una variable inédita que puede contribuir en alguna medida al desarrollo conceptual y empírico, respecto a la procrastinación académica.

Asimismo, a futuro se pretende realizar un estudio a fondo de carácter cualitativo, donde el investigador exponga su cosmovisión del fenómeno en el contexto y puedan surgir probables 
modelos, que permitan comprender e interpretar esa realidad de un modo menos generalizado y por tanto, más útil para los miembros de la comunidad universitaria del estudio.

\section{CONCLUSIONES}

Finalizado el estudio, se puede mencionar que la fecha límite, es un factor de importancia considerado por los alumnos, ya que a pesar de haberse asignado todas las tareas en la primera semana de clases y que las mismas no requerían conocimientos previos, ni de los cursados en el período de la investigación; más del 70\% en promedio de los estudiantes, realizó las entregas en tal fecha. Lo anterior, al ser confrontado con entrevista y cuestionario determinó que la mayoría las realizaba dos días o menos antes del límite; en este sentido, más de la mitad de los casos relacionaron la demora a situaciones asociadas a la tecnología.

Para poder determinar la procrastinación académica de las tareas, un elemento clave más allá de la fecha límite de entrega, es el tiempo de antelación con que se realiza la misma, pues como se mencionó, muchos de los que la entregan en el límite, la realizan incluso el día anterior. Este tiempo, se obtuvo a través de una entrevista, lo que pudiera llevar a pensar en un sesgo al respecto de lo reportado por los estudiantes; sin embargo, escalas calificadas como la Escala de Procrastinación Académica (EPA), avalada en varios países latinoamericanos, tendrían el mismo sesgo pues obtiene esta información, a partir de la afirmación dicotómica siguiente "Generalmente me preparo por adelantado para los exámenes" cuya resultado de igual forma, está expuesto al sesgo representado por la respuesta del estudiante. Similar situación se presenta para la escala Tuckman, que consigue esta información con la afirmación dicotómica "Cuando tengo una fecha límite, espero hasta el último minuto".

Se pudo verificar, que existe presencia de procrastinación académica en la mayoría de la población, aunque en muchos casos la misma entra en la clasificación de lo que se conoce como procrastinación activa, lo que surge como vínculo para investigaciones específicas respecto a este tipo de procrastinación. Los resultados obtenidos, son específicos para el área de la investigación (Física), siendo esta moderadora en cuanto al fenómeno de estudio. Resultados distintos se podrían obtener con otras áreas como por ejemplo electrónica, donde existe un mayor manejo instrumental y sus contenidos son más prácticos, también en asignaturas llamadas por los estudiantes en las entrevistas como "las teóricas", que son las que no requieren de cálculo y a las que se refirieron como de "relleno".

Una de las circunstancias más reportadas en las entrevistas sobre el comportamiento procrastinador, fue que los alumnos perciben que funcionan mejor "bajo presión". Los resultados respecto a la fecha límite y su complemento por medio de la entrevista, efectivamente mostraron que su comportamiento va de acuerdo a esa percepción, lo cual sería una variable interesante de medir para futuras investigaciones en la misma población.

Del mismo modo, se observó que el tiempo dedicado al estudio durante el tiempo libre dentro de las instalaciones de la universidad, resultó una variable inédita dentro del campo de la procrastinación académica, lo que resultó uno de los aportes más importantes del trabajo, el cual puede ser profundizado, ya que no se encontraron estudios relacionados con la variable. 


\section{REFERENCIAS BIBLIOGRÁFICAS}

Albarrán, J. (2019). La deserción estudiantil en la Universidad de Los Andes (Venezuela). Revista Educación y humanismo, 21(36), 60-82. http://dx10.17081/eduhum.21.36.2806

Arias, F. (2016). El proyecto de investigación (Séptima ed.). Episteme.

Barraza, A., \& Barraza, S. (2018). Evidencias de validez y confiabilidad de la escala de procrastinación académica en una población estudiantil mexicana. Revista de Psicología y Ciencias del Comportamiento, 9(1), 75-99. http://www.scielo.org.mx/pdf/rpcc/v9n1/20071833-rpcc-9-01-75.pdf

Barreto, M. (2015). Relajación en estados de ansiedad y procrastinación en ingresantes de la facultad de ciencias sociales. Tesis Doctoral, Lima. https://cybertesis.unmsm.edu.pe/bitstr eam/handle/20.500.12672/4543/Barreto_em.pdf?sequence=1\&isAllowed=y

Bermúdez, K. (2020). La procrastinación en el desarrollo profesional del docente universitario. Tesis de Maestría, Universidad de Carabobo, Valencia. http://www.riuc.bc.uc.edu.ve/hand le/123456789/8472

Castro, S., \& Mahamud, K. (2017). Procrastinación académica y adicción a internet en estudiantes universitarios de Lima Metropolitana. Av.psicol, 189-197. https://www.unife.edu.pe/publi caciones/revistas/psicologia/2017_2/PROCRASTINACI\%C3\%93N.pdf

Chu, A. H. C., \& Choi, J. N (2005). Rethinking Procrastination: Positive Effects of "Active" Procrastination Behavior on Attitudes and Performance. The Journal of Social Psychology, 145(3), 245-264. https://doi.org/10.3200/SOCP.145.3.245-264

Cirino, G. (2003). Los intereses como motivación intrínseca en la sala de clases. Perspectivas psicológicas, 80-84. http://pepsic.bvsalud.org/pdf/pp/v3-4/v3-4a08.pdf

Condori, Y., \& Mamani, K. (2016). Adicción al Facebook y procrastinación académica en estudiantes de la Facultad de Ingeniería y Arquitectura de la Universidad Peruana Unión. Tesis de grado de Psicología, Universidad Peruana Unión, Juliaca, Perú. https://repositorio.upeu.edu.pe/bitstream/handle/UPEU/177/Yuli_Tesis_bachiller_2016.p df? sequence $=1 \&$ is Allowed $=\mathrm{y}$

Coronado, G. (2015). Procrastinación académica y estrategias de afrontamiento en estudiantes de maestría de educación mención investigación educativa. Universidad Nacional Experimental Rómulo Gallegos. San Juan de los Morros. Estado Guárico. Tesis de pregrado, Universidad Bicentenaria de Aragua, San Joaquin de Turmero.

Díaz-Morales, J. (2019). Procrastinación: Una Revisión de su Medida y sus Correlatos. Revista Iberoamericana de Diagnóstico y Evaluación - e Avaliação Psicológica, 51(2), 43-60. https://doi.org10.21865/RIDEP51.2.04 
Domínguez, S., \& Campos, Y. (2017). Influencia de la satisfacción con los estudios sobre la procrastinación académica en estudiantes de psicología: un estudio preliminar. Liberabit, 23(1), 123-135. https://doi.org/10.24265/liberabit.2017.v23n1.09

Galarza, C., Jadán, J., Paredes, L., Bolaños, M., \& Gómez, A. (2017). Procrastinación, adicción al internet y rendimiento académico de estudiantes universitarios ecuatorianos. Estudios Pedagógicos, XLIII(3), 275-289. https://doi.org/10.4067/S0718-07052017000300016

García, C. (2009). Comprendiendo la Procrastinación con el modelo ABC de Albert Ellis. Gaceta de la escuela de medicina Justo Sierra, 2(1), 4-5. https://www.researchgate.net/publication

/264421119_Comprendiendo_la_procrastinacion_con_el_Modelo_ABC_de_Albert_Ellis /link/53dd8f6f0cf216e4210c1d57/download

Garzón, A., \& Gil, J. (2017a). Gestión del tiempo y procrastinación en la educación superior. Universitas Psychologicas, 16(3), 1-13.https://doi.org/10.11144/Javeriana.upsy16-3.gtpe

Garzón, A., \& Gil, J. (2017b). El papel de la procrastinación académica como factor de la deserción unversitaria. Revista Complutense de Educación, 28(1), 307-324. http://dx.doi.org/10.5209 /rev_RCED.2017.v28.n1.49682

Gil, J., Besa De, M., \& Garzón, A. (2020). ¿Por qué procrastina el alumnado universitario? Análisis de motivos y caracterización del alumnado con diferentes tipos de motivaciones. Revista de Investigación educativa, 38(1), 183-200. doi:DOI: http://dx.doi.org/10.6018/rie.344781

González, L., \& Tovar, R. (2015). Procrastinación académica en estudiantes de la facultad de ingeniería. Trabajo especial de grado, Universidad Rafael Urdaneta, Maracaibo.

Hernández, R., Fernández, C., \& Baptista, P. (2014). Metodología de la Investigación. (Quinta ed.). Mc Graw Hill.

Hurtado, J. (2015). El proyecto de investigación (Octava ed.). Quirón Ediciones.

Matalinares, M., Ornella, R., Díaz, A., Baca, D., Rivas, L., \& Uceda, J. (2017). Procrastinación y adicción a redes sociales en estudiantes universitarios de pre y post grado de Lima. Horizonte de la ciencia, 7(13), 63-81. https://doi.org/10.26490/uncp.horizonteciencia.2017

\section{.13 .355}

Morales, A. (2019). La procrastinación académica desde la cosmovisión del estudiante universitario, centrada en sus sistemas sociales. Tesis doctoral, Universidad Pedagógica Experimental Libertador, Maracay.

Natividad, L. (2014). Análisis de la procrastinación en estudiantes universitarios. Tesis doctoral, Universitat de València, Valencia. http://roderic.uv.es/bitstream/handle/10550/37168/Tesi 
s\%20Luis\%20A.\%20Natividad.pdf?sequence=1\&isAllowed=y

Naturil, C., Peñaranda, D., Vicente, J., \& Marco, F. (2018). Mala gestión del tiempo en los estudiantes universitarios: efectos de la procrastinación. Congreso Nacional de Innovación Educativa $y$ de Docencia en Red. Valencia. http://dx.doi.org/10.4995/INRED2018.2018.88

Neenan, M. (2008). Tackling Procrastination: An REBT Perspective for Coaches. Journal of Rational-Emotive and Cognitive-Behavior Therapy, 26(1), 53-62. 10.1007/s10942-0070074-1

Onwuegbuzie, A. (2004). Academic procrastination and statistics anxietu. Assessment \& Evaluation in Higher Education, 29(1), 3-19. https://doi.org/10.1080/02602930420001603 84

Real Academia Española. (2014). https://dle.rae.es/procrastinar

Rodríguez, A., \& Clariana, M. (2017). Procrastinación en Estudiantes Universitarios : Su Relación con la Edad y el Curso Académico. Revista Colombiana de Psicología, 26(1), $45-$ 60.10.15446/rcp.v26n1.53572

Rodríguez, M. (2013). La procrastinación. Rompan Filas, 11-19. Obtenido de https://ityc.edu.mx/ wp-content/uploads/2019/01/123-RompaA\%E2\%80\%A2v9_Finala.pdf

Rothblum, E. (1990). Fear of failure: the psicodynamic need archievement, fear of success and procrastination models. Handbook of social and evaluation anxiety, 497-537. https://doi.org/10.1007/978-1-4899-2504-6_17

Schraw, G., Wadkins, T., \& Olafson, L. (2007). Doing the Things We Do A Grounded Theory of Academic Procrastination. Journal of Educational Psychology, 99(1), 12-25. 10.1037/0022-0663.99.1.12

Somers, P. (2008). Gênero e outras variáveis que influenciam na procrastinação acadêmica. Revista Educação, 31(1), 54-60/. http://revistaseletronicas.pucrs.br/ojs/index.php/faced/art icle/view/2758

Steel, P. (2011). Procrastinación. ¿Por qué dejamos para mañana lo que podemos hacer hoy? Grijalbo.

Steel, P., \& Klingsieck, K. (2016). Academic procrastination: psychological antecedents revisited. Australian Psychologist, 51(1), 36-46. https://doi.org/10.1111/ap.12173 
Tarazona, F., Aliaga, I., Romero, J., \& Veliz, M. (2016). Procrastinación académica en estudiantes de educación en Lenguas, Literatura y Comunicación: Características, modos y factores. Horizonte de la ciencia, 6(10), 185-194. https://www.redalyc.org/articulo.oa?id=5709608 70018

Thorndike, E. (1911). Animal Intelligence.: The Macmillan Company. https://openlibrary.org/boo ks/OL7172495M/Animal_intelligence

Tuckman, B. (2002). The relationship of academic procrastination, rationalizations and performance in a web course with deadlines. Annual Meeting of the American Psychological Association. Chicago. https://files.eric.ed.gov/fulltext/ED470567.pdf

Conflicto de intereses / Competing interests:

El autor declara que no incurre en conflictos de intereses.

Rol de los autores / Authors Roles:

No aplica.

Fuentes de financiamiento / Funding:

El autor declara que no recibió un fondo específico para esta investigación.

Aspectos éticos / legales; Ethics / legals:

El autor declara no haber incurrido en aspectos antiéticos, ni haber omitido aspectos legales en la realización de la investigación. 